\title{
THE CORRELATION BETWEEN REACTIVE OXYGEN SPECIES AND ANTIOXIDANTS
}

\author{
ALINA OANA RUSU (MOLDOVAN) ${ }^{\mathrm{a}, \mathrm{b}}$, MARIA IULIANA GRUIA ${ }^{\mathrm{c}^{*}}$, \\ VIORICA LAZAR LORDEAN ${ }^{d}$, DAN MIHU ${ }^{b}$
}

\begin{abstract}
Oxidative stress represents as an important factor in carcinogenesis and may play a role in initiation and progression of tumors. Oxidative stress is responsible for DNA damage, and includes a multitude of lesions, many of which are mutagenic and have multiple roles in cancer and aging. The aim of the study is to demonstrate that an antioxidant treatment administration before surgery can influence the breast cancer patients' immune response.Whole blood samples from 113 patients with breast cancer, admitted in the Surgery Department III of the Oncology Institute of Bucharest were collected and the blood serum was isolated, and then made determinations of malondialdehyde, ceruloplasmin, albumin thiols, and total antioxidants. What was shown in this study is that ROS can increase cell proliferation and genomic instability, which stimulates disease progression, and the tumor is an oxidative stress inducer that can influence the redox balance. The key of an effective compensatory body response to this imbalance, cannot be done without exogenous antioxidant support.
\end{abstract}

Keywords: ROS, breast cancer, oxidative stress mechanisms, antioxidants

\section{INTRODUCTION}

The interest in a more accurate understanding and deepening the underlying oxidative stress mechanisms and its implications in various

\footnotetext{
a Department of Surgery III, "Prof. Dr. Alexandru Trestioreanu" Institute of Oncology, Bucharest, 252 Fundeni Road, Bucharest, Romania

b Department of Obstetrics and Gynecology II, "Iuliu Haţieganu" University of Medicine and Pharmacy,55-57 21 December 1989 Bulevard,Cluj-Napoca

" Department of Biochemistry and Radiobiology, "Prof. Dr. Alexandru Trestioreanu" Institute of Oncology, 252 Fundeni Road, Bucharest, Romania

'Department of Medicine II, "Vasile Goldis" University of General Medicine, 86 Liviu Rebreanu Street, Arad, Romania

*Corresponding author: gmariaiuliana@yahoo.com
} 
neoplasms has become very high in the recent decades. To prevent cell damage by oxidative stress, the aerobic body cells have developed over time a series of extremely powerful anti-oxidant strategies.

Antioxidants are compounds with the ability to reduce ROS and are classified into endogenous and exogenous, enzymatic and non-enzymatic antioxidants.

Among the primary important endogenous antioxidants, we can mention the enzymatic ones (superoxide dismutase, catalase and glutathione peroxidase) or the non-enzymatic ones (vitamin $\mathrm{E}, \mathrm{C}$ ) while the exogenous ones are chemical compounds with polyphenolic structures, anthocyanins, etc. [1]

A<smiles>Cc1c(C)c2c(c(C)c1O)CCC(C)(C(C)(C)CCO)O2</smiles>

Vitamin E, TOH

reactive

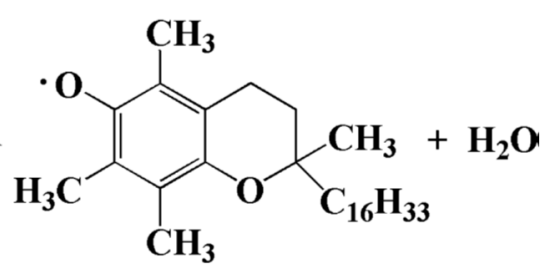

TO·, less reactive

B<smiles></smiles>

Vitamin C, AscH $^{-}$reactive<smiles>O=C1OC(C(O)CO)C(=O)C1=O</smiles>

Asc $^{-\cdot}$, stable

C $\mathrm{AscH}^{-}+\mathrm{TO} \longrightarrow \mathrm{TOH}+\mathrm{Asc}^{-}$

Figure 1. Direct reactions of vitamin $\mathrm{E}(\mathrm{TOH})$ with $\cdot \mathrm{OH}(\mathrm{A})$ and vitamin $\mathrm{C}(\mathrm{AscH})$ with $\mathrm{ROO}(\mathrm{B})$ and regeneration of vitamin $\mathrm{E}$ from vitamin $\mathrm{C}(\mathrm{C})$.

Antioxidants can be molecules that can neutralize free radicals by accepting or donating electrons to eliminate the unpaired state of the radical. Antioxidant molecules can directly react with reactive radicals and destroy them, while new free radicals can become less active, have a longer lifespan and be less dangerous than those radicals they have neutralized. They can be neutralized by other antioxidants or other mechanisms to end their radical status. Many antioxidants have aromatic ring structures and are able to 
relocate unmatched electrons (Fig.1). Vitamin $\mathrm{C}(\mathrm{AscH})$ in the aqueous phase and vitamin $E(T O H)$ in the lipid phase will react directly with or will neutralize the hydroxyl, alkoxyl and lipid peroxyl (ROO $\cdot$ ) radicals and form $\mathrm{H}_{2} \mathrm{O}$, alcohol and lipid hydroperoxides.

Vitamin $E$ itself becomes a phenyl radical, and vitamin $C$ will become into a very stable radical (Asc •), due to its delocalized structure (Fig. $1 A$ and $B$ ). In addition, vitamin $\mathrm{C}$ can also neutralize the radical form of other antioxidants such as the glutathione radical and vitamin $\mathrm{E}$ radical and regenerate them (Fig. 1C). Vitamin $\mathrm{C}$ itself is easily regenerated into Asc - with $\mathrm{NADH}$ or NADPH dependent reductase [2].

In case of increased oxidative stress, free radicals can cause cytotoxicity, inhibit cell proliferation and have an important role in inducing cell death by apoptosis or necrosis.

Medium or low oxidative stress can induce cellular mutations [3]. It is well known that in high concentrations, ROS have a destructive action at the cellular level, but in low concentrations they can act as signaling molecules.

Once reactive oxygen species are formed, they are rapidly broken down in by the cells' endogenous antioxidant systems.

Recent studies show that in the breast cancer microenvironment, oxidative stress causes mitochondrial dysfunction at the subcellular level. This is manifested by regulating several factors, such as nuclear respiratory factor 1 (NRF1), which increases in the tumor tissue due to the installed oxidative stress [4].

Reactive oxygen species (ROS) are produced by both enzymatic and non-enzymatic systems in eukaryotic cells and play important roles in the cells' physiology and pathophysiology. Although physiological concentrations are essential for cell survival, overproduction of ROS is detrimental in normal cells and is considered a key factor in the onset of several diseases, such as neurodegenerative diseases, cardiovascular disorders and cancer [5].

Increased production of reactive oxygen species has been shown in many cancers, but their involvement in carcinogenesis and tumor progression is not yet fully understood. High ROS levels are oncogenes, that can cause DNA damage, promote genetic instability and tumorigenesis, act as signaling molecules, cause tumor cell proliferation and survival. They also stimulate abnormal cell growth, invasion and metastasis, and produce resistance to apoptosis $[6,7,8]$.

Reactive oxygen species interact with essential macromolecular structures, especially with nucleic acids, proteins and lipids. Because these structures are essential for the tumor metabolism, ROS interferes with the normal cell function [9]. 
The aim of the study is to demonstrate that an antioxidant treatment administration before surgery can influence the breast cancer patients' immune response.

\section{RESULTS AND DISCUSSION}

The patients' clinical condition was assessed by a complete clinical examination and the performance status was assessed using the ECOG scale.

At diagnosis, $72.6 \%$ of patients $(82 / 113)$ presented a good performance status ECOG 0 (Fully active), 24.8\% (28/113) with ECOG 1 (able to perform normal light or sedentary physical activities) and 2.6\% (3/113) with ECOG 2 (mobile, apparently active, but unable to work over $50 \%$ of their physically active time) (Fig. 2).

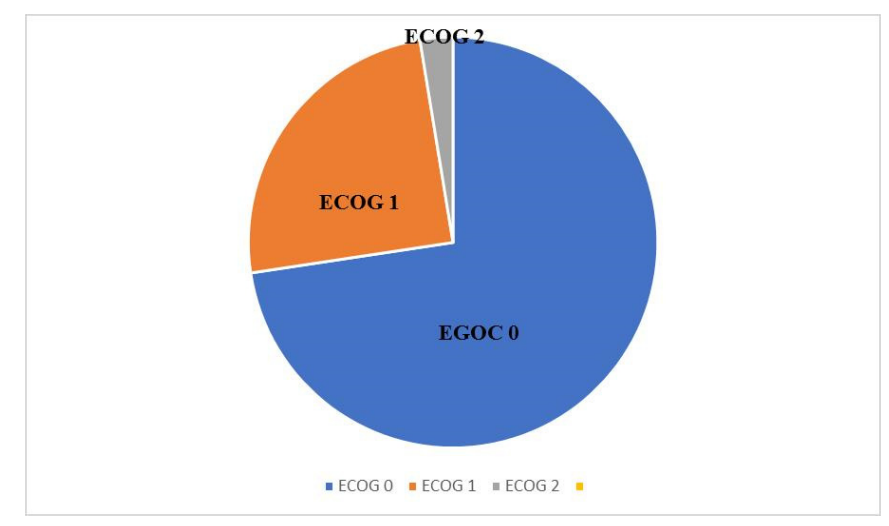

Figure 2. Representation of patients' performance status.

In the studied group, the staged distribution of the disease was heterogeneous: stage IIA - IIB and IIIA, with $80 \%$ invasive ductal carcinomas: solid tumors, imprecisely delimited, hard consistency, firm, because of the calcifications and desmoplastic reaction.

The remaining $20 \%$ patients presented lobular carcinomas with a discrete, firm form, a grey-white mass with irregular borders. The background stroma is densely fibrous and contains foci with periductal and perivascular elastosis, with or without peritumoral lymphocytic infiltrate.

The patients' elective surgical indication was radical mastectomy. All the patients received premedication and standardized anesthesia and were admitted to intensive care after surgery. 
For the investigated group, the obtained average values of the oxidative stress parameters are presented in the table below. There is a statistically significant increase of all registered parameters compared to the normal value (Table 1). For each patient were taken four blood samples, in dynamic: 24 hours before surgery, at the time of the anesthesic administration, 24 hours after surgery and at 7 days after surgery.

Table 1. The average values of the oxidative stress parameters

\begin{tabular}{|l|r|r|r|r|}
\hline & $\begin{array}{c}\text { Malondialdehyde } \\
(\mu \mathrm{mol} / \mathbf{1 0 0} \mathbf{~ m l} \\
\text { ser) }\end{array}$ & $\begin{array}{c}\text { Cerulo- } \\
\text { plasmin }\end{array}$ & \multicolumn{1}{|c|}{ Thiols } & Antioxidants \\
\hline Average & 6.84 & 164.51 & 383.14 & 1.36 \\
\hline $\begin{array}{l}\text { Standard median } \\
\text { error }\end{array}$ & 0.20 & 4.1 & 8.89 & 0.03 \\
\hline Median & 6.35 & 169 & 325 & 1.34 \\
\hline Standard deviation & 1.94 & 62.84 & 132.11 & 0.49 \\
\hline Minimum & 3.16 & 65 & 183 & 0.06 \\
\hline Maximum & 10.83 & 264,02 & 583.28 & 4.10 \\
\hline Normal value & $0-4 \mu \mathrm{mol} / 100 \mathrm{ml}$ & $80-120 \mathrm{U} . \mathrm{I}$ & $370-450 \mu \mathrm{mol} / \mathrm{l}$ & $0.9-1.4 \mu \mathrm{mol} / \mathrm{l}$ \\
\hline
\end{tabular}

Regarding the dynamic evolution of the lipid peroxidation, it is found that the value of malondialdehyde decreased after the anesthetic-surgical time. The mean value recorded at the first administration was $8.95 \mu \mathrm{mol} /$ $100 \mathrm{ml}$, much higher than the normal maximum value of $4 \mu \mathrm{mol} / 100 \mathrm{ml}$ decreasing to $7.07 \mu \mathrm{mol} / 100$. All ceruloplasmins' values remained relatively constant, the initial value being 176.04 U.I., and at the end registering the value of 199.29 U.I. (Fig. 3).
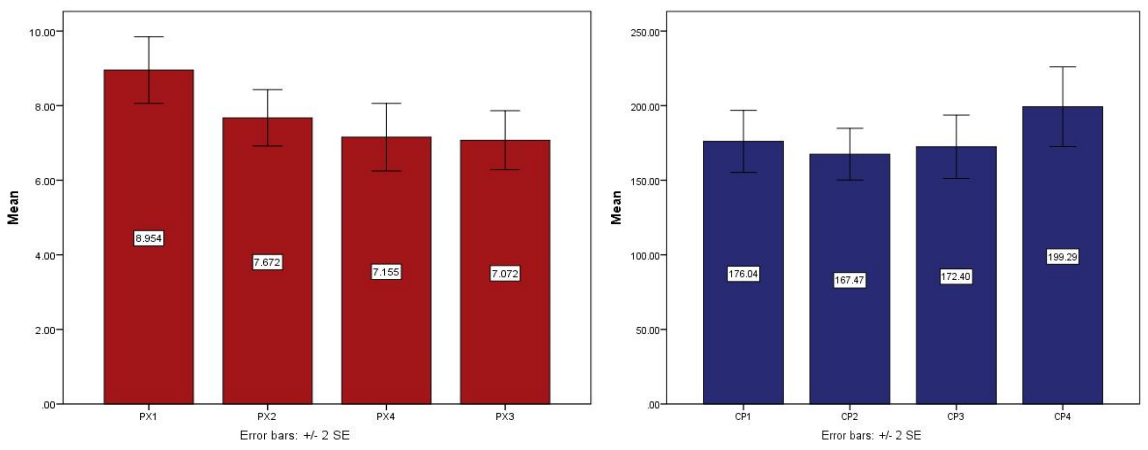

Figure 3. Evolution in dynamics of lipid peroxidation by determining the values of malondialdehyde and ceruloplasmin 

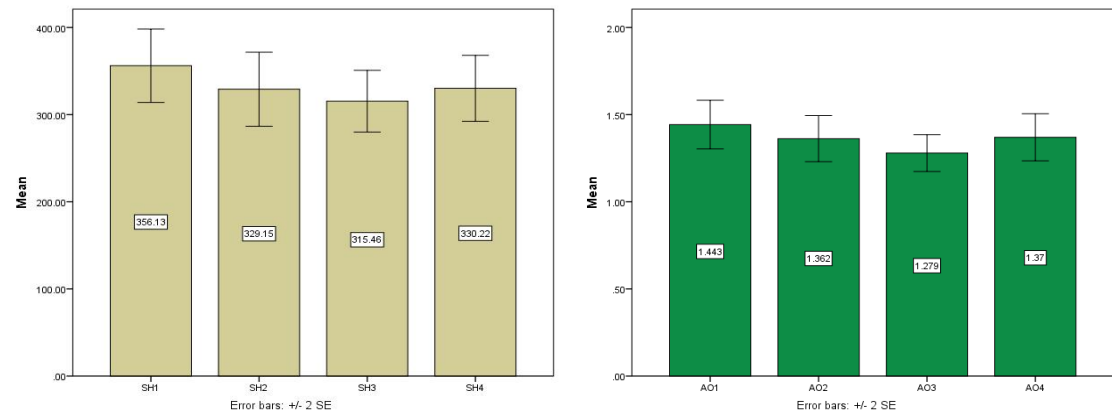

Figure 4. Dynamic evolution of albumin thiols and changes in dynamic of the average values of the total antioxidants

Albumin thiol values also decreased after the surgical treatment from $357.18 \mu \mathrm{mol} / \mathrm{I}$ to $330.21 \mu \mathrm{mol} / \mathrm{I}$.

The dynamics of antioxidants was similar with the thiols, so that the average value at the first determination was $1.44 \mu \mathrm{mol} / \mathrm{I}$, and finally 1.37 $\mu \mathrm{mol} / \mathrm{I}$ (Fig. 4).

The presented data claim that the oxidative stress balance is more inclined in the favor of free oxygen radical excess production in the detriment of their uptake. The defense mechanisms represented by antioxidants can't compensate for the fact that the tumor is an inducer of oxidative stress (Fig. 5).
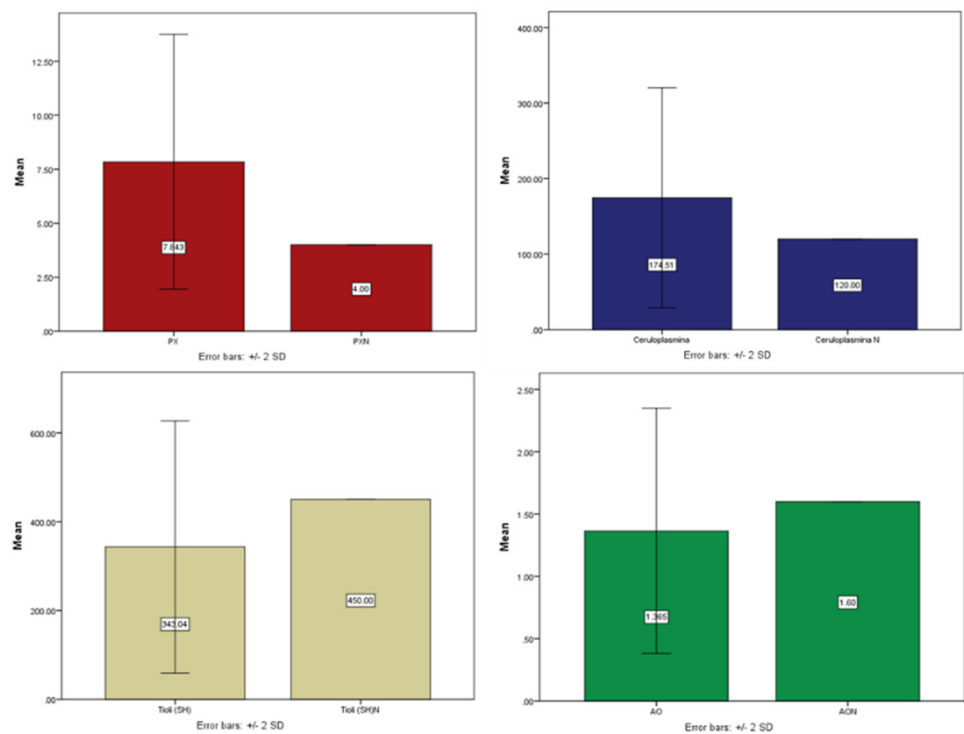

Figure 5. Average values of malondialdehyde, ceruloplasmin, thiols and antioxidants 
In our study group, it was found that there is a very strong correlation between lipid peroxidation values determined by measuring malondialdehyde and ceruloplasmin values $(r=0.382, p<0.0001)$. There are statistically significant correlations between the values of ceruloplasmin and albumin thiols $(r=0.260, p<0.0001)$ (Fig. 6A and Fig. 6B).
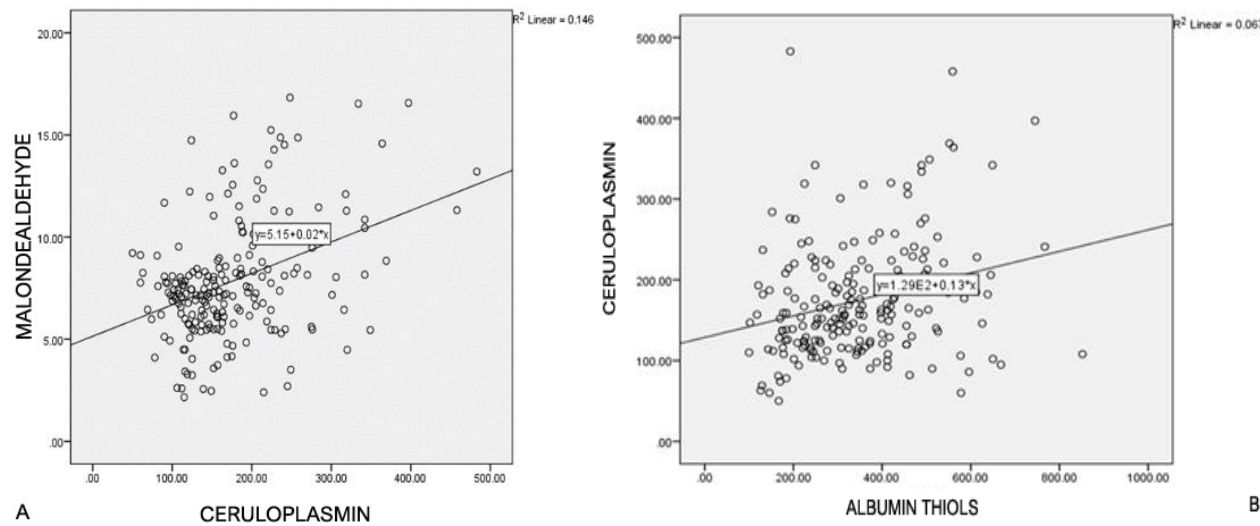

Figure 6A. Correlation between lipid peroxidation values represented by malondialdehyde and ceruloplasmin values; 6B. Correlation between ceruloplasmin and thiol values

In the group of patients studied there was a strong correlation between the values of albumin thiols and the value of the total antioxidants $(r=0.196, p<0.005)$, as a direct correlation also exists between lipid peroxidation values (default of malondialdehyde) and total thiols $(r=0.297$, $p<0.0001$ ) (Fig. 7A and Fig. 7B).

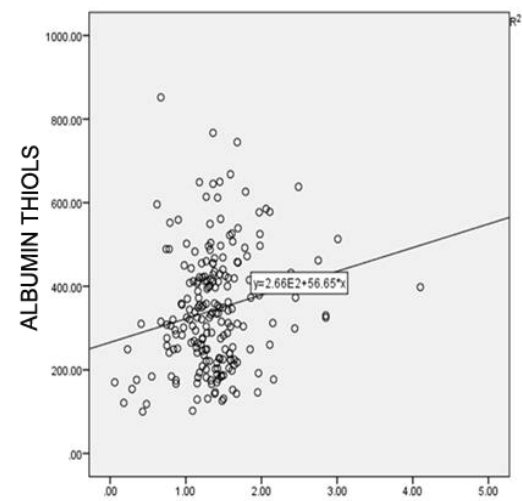

A TOTAL ANTIOXIDANTI

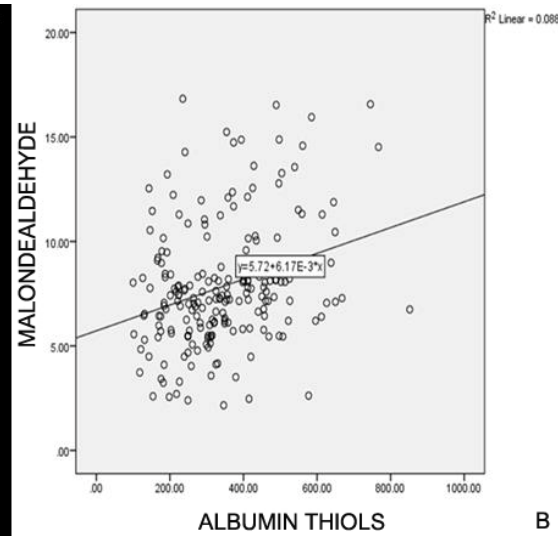

B

Figure 7A. Correlation between albumin thiol values and total antioxidant values; 7B. Correlation between the value of malondialdehyde and the value of thiols 
Statistically, there were no significant correlations between the values of malondialdehyde and antioxidants $(r=0.0380$ and $p<0.05)$ and between the values of ceruloplasmin and antioxidants $(r=0.984, p>0.001)$ (Fig. $8 \mathrm{~A}$ and Fig. 8B).
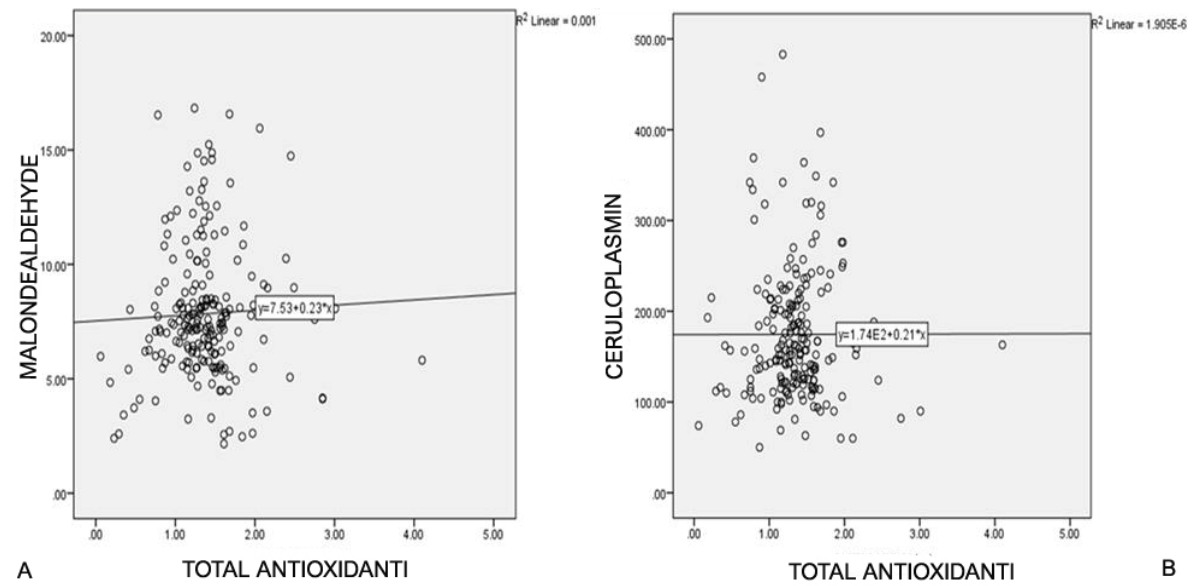

Figure 8A. Correlation between the value of malondialdehyde and antioxidants;

8B. Correlation between the value of ceruloplasmin and antioxidants

To prevent cell damage by oxidative stress, the cells of aerobic organisms have developed over time a series of extremely powerful anti-oxidant strategies. ROS may be accidentally generated as a consequence of altered cellular metabolism (eg during cellular respiration cicle), or by inflammation.

ROS have oxygen-derived molecules that are thought to be responsible for severe metabolic imbalances [10]. The main source of intracellular ROS is determined by the oxidative process in the mitochondrial respiratory chain. Normally in a healthy body oxidative stress reacts as a signal. But under pathophysiological conditions, it is a determining factor in malignant cell amplification and phenotyping [11].

Martindale and Holbrook estimated that ROS occurs in 1-2\% of the oxygen used by mitochondria during oxidative phosphorylation and is therefore considered to be a normal by-product of the metabolism energy. Some common forms of ROS resulting from oxidative phosphorylation like superoxide anion $\left(\mathrm{O}_{2} \bullet\right)$, such as hydrogen peroxide $\left(\mathrm{H}_{2} \mathrm{O}_{2}\right)$ and hydroxyl radicals $(\mathrm{OH} \bullet)$, act as oxidants, stealing electrons from nucleic acids, proteins and lipids. Normally the body is accustomed to elevated levels of ROS by activating the apoptotic pathways, and mechanisms that convert ROS to non-toxic products are endogenous absorption enzymes (e.g., antioxidants) [12]. 
ROS interferes with the body through two effects: primary and secondary. The primary effect is localized and reversible, depending on the intensity of the oxidative stress and the level of antioxidants and highlighted by a performance score $0-1$ (changes in cell membrane permeability, ionic imbalances, peroxidation of fatty acids in cell membranes, changes in cellular metabolic processes because of protein alterations and the decreased energy production).

The transition to the secondary effect marks the irreversible phase and is accompanied by the appearance of clinical manifestations like cell lysis, altered biofunctions, low activity resistance, or to infections - specific for a 2-4 score. In this stage the imbalance of calcium ions that promotes the activation of hydrolytic enzymes, protease, cell lysis, vascular disorders, peroxidation of proteins and nucleic acids, altered immune reactivity, tissue damage located in one or more organs. High exercise activity causes oxidative stress only in overload training (in large volume or long-term period) and can lead to an impaired antioxidant defense [13].

Saed, Diamond and Fletcher $N$. in their paper established that in the cancer cell, the presence of the imbalance between the production of reactive oxygen species and the elimination of free radicals leads to a state of oxidative stress and to the destruction of some essential components of the cell [14].

M. Ryan Smith, Praveen K. Vayalil, Fen Zhou, Gloria A. Benavides in their work they demonstrated that free oxygen radicals released by the malignant tumor can be partially but not totally inhibited by the administration of antioxidants, as demonstrated by the determination of thiols and other components in their study [15].

he data presented suggest that the balance of oxidative stress is more inclined in the favor of the excess production of free oxygen radicals to the detriment of their uptake.

Weinberg and Chandel demonstrated that elevated ROS levels overwhelm the cell's ability to respond appropriately. ROS overproduction becomes a threat to nucleic acids, proteins and lipids that ultimately lead to oncogenic transformation [16].

\section{CONCLUSIONS}

The defense mechanisms represented by antioxidants cannot compensate for the fact that the tumor is an inducer of oxidative stress. The disease progression is represented by resistance to apoptosis, increased cellular mobility with the increased metastatic capacity, as well as the 
conversion to anaerobic glycolysis. On the other hand, the excess production of ROS is in the detriment of cancer cell survival and proliferation, through the destructive mechanisms given by the oxidative-destructive reactions of biomacromolecules essential to life.

All of the determinations strengthen the idea that ROS overproduction and the lack of an effective compensatory body response to this imbalance, cannot be done without exogenous antioxidant support.

\section{EXPERIMENTAL SECTION}

There were studied in dynamic 113 patients with breast cancer, admitted in the Surgery Department III of the Oncology Institute of Bucharest for surgery treatment, between October 2016 and December 2018.

The patients were diagnosed with breast cancer, the results firmly confirmed by histopathological reports.

The approval of the study was obtained from the Ethics Committee of the Oncology Institute of Bucharest No. 15920/12.11.2018.

After giving their personal consent in writing, whole blood samples were collected by venous puncture. By centrifugation using a Beckman J2-J1 model (Germany, 2002), the blood serum was isolated, and then the following determinations were made:

- The lipid peroxides were evaluated by measuring the final concentration of malondialdehyde (MDA). The method is spectrophotometric and is based on the formation of a colored adduct (MDA- TBA2) that develops maximum absorption at $532 \mathrm{~nm}$ depending on the concentration.

- The oxidative activity of ceruloplasmin. We used the Ravin method by reaction with $\mathrm{p}$-phenylenediamine in acetic acid-acetate pad. The color intensity developed at $540 \mathrm{~nm}$ is directly proportional with the concentration of the measured compound.

- The thiol-albumin groups TA were determined by reaction with the 5,5'-dithiobis (2- nitrobenzoic) acid, Ellman's reagent, which, following oxidations, develops the maximum intensity at $412 \mathrm{~nm}$ in accordance with the concentration of the $\mathrm{SH}$ groups formed.

- The total antioxidants were biochemically measured by a method that utilizes the ability of the serum to reduce iron. When the $\mathrm{pH}$ is low, the Fellltripyridyl-s-triazine complex (Felll -TPTZ) is reduced to its ferrous form, by forming a new complex that develops an intense blue color, measurable, with a maximum absorption at $593 \mathrm{~nm}$.

In order to make these determinations, the solutions were prepared using compounds of analytical purity from Merck and Sigma, the water that 
has been used was genetically pure after filtering by Millipore devices (MilliQ-Biocel, United States, 2010), and the spectrophotometric readings were carried out on a Specord 210 spectrophotometer (Germany 2005).

\section{ACKNOWLEDGMENTS}

No funding was received for this work. This research did not receive any specific grant from funding agencies in the public, commercial, or notfor-profit sectors.

\section{REFERENCES}

1. A.R. Nourazarian, P. Kangari, A. Salmaninejad; Asian Pac J Cancer Prev., 2014, 15, 4745-4751.

2. M.A. Hossain, K. Asada; J Biol Chem, 1985, 260, 12920-6.

3. I.I.C. Chio, D.A.Tuveson; Trends in Mol Med, 2017, 23, 411-29.

4. R.C. Rose, A.M. Bode; Biochem. J, 1995, 306, 101-105.

5. V. Adler, Z. Yin, K.D. Tew, Z. Ronai; Oncogene, 1999, 18, 6104-6111.

6. L. Gate, J. Paul, G.N. Ba, K.D. Tew, H. Tapiero; Biomedicine \& Pharmacotherapy, 1999, 53, 169-180.

7. D.H. Kang; AACN Clinical Issues, 2002, 13, 540-549.

8. G. Sigounas, A. Anagnostou, M. Steiner; Nutrition and Cancer, 1997, 28, 30-35.

9. S. Kiokias, M.H. Gordon; European Journal of Clinical Nutrition, 2003, 57, 11351140.

10. S. Sharhar, H. Normah, A. Fatimah, R.N. Fadilah, Ga. Rohi, et al; Asian Pacific Journal of Cancer Prevention, 2008, 9, 343-349.

11. R. Kumaraguruparan, S. Naghini, et al; Clinica Chimica Acta, 2002, 325, 165-170.

12. J.L. Martindale, N.J. Holbrook; J Cell Physiol, 2002, 192(1), 1-15.

13. E. Mihalas, LI. Serban, D. Matei, D. Cascaval, Al. Galaction; Studia UBB Chemia, 2019, LXIV (2), Tom I, 35-47.

14. G.M. Saed, M.P. Diamond, N.M. Fletcher; Gynecol Oncol., 2017, 145(3), 595-602.

15. M.R. Smith, P.K. Vayalil, F. Zhou, G.A. Benavides, et al; Redox Biol., 2016, 8, 136-48.

16. F. Weinberg, N.S. Chandel; Ann N Y Acad Sci., 2009, 1177, 66-73. 